\title{
ARTICLE OPEN Printed microelectrode arrays on soft materials: from PDMS to hydrogels
}

\author{
Nouran Adly $\mathbb{D}^{1,2}$, Sabrina Weidlich ${ }^{1}$, Silke Seyock ${ }^{1}$, Fabian Brings ${ }^{1}$, Alexey Yakushenko ${ }^{1}$, Andreas Offenhäusser $\mathbb{D}^{1}$ and \\ Bernhard Wolfrum ${ }^{1,2}$
}

Microelectrode arrays (MEAs) provide promising opportunities to study electrical signals in neuronal and cardiac cell networks, restore sensory function, or treat disorders of the nervous system. Nevertheless, most of the currently investigated devices rely on silicon or polymer materials, which neither physically mimic nor mechanically match the structure of living tissue, causing inflammatory response or loss of functionality. Here, we present a new method for developing soft MEAs as bioelectronic interfaces. The functional structures are directly deposited on PDMS-, agarose-, and gelatin-based substrates using ink-jet printing as a patterning tool. We demonstrate the versatility of this approach by printing high-resolution carbon MEAs on PDMS and hydrogels. The soft MEAs are used for in vitro extracellular recording of action potentials from cardiomyocyte-like HL-1 cells. Our results represent an important step toward the design of next-generation bioelectronic interfaces in a rapid prototyping approach.

npj Flexible Electronics (2018)2:15; doi:10.1038/s41528-018-0027-z

\section{INTRODUCTION}

Microelectrode arrays (MEAs) have attracted strong interest due to their use in various applications, including cellular recording, biosensors, and drug screening. ${ }^{1-11}$ Perhaps one of the most promising application of MEA devices are biomedical implants in which the MEA serves as a vital tool for monitoring or restoring biological functionality. ${ }^{12-14}$ Historically, MEA devices, as developed by Wise and co-worker ${ }^{15}$ in the late 1960s, consisted of conductive metallic material covered by an insulating layer, except for a small electrode opening to establish a connection to the surrounding tissue. They were fabricated on stiff silicon substrates using photolithographic techniques and silicon etching technology. These and similar devices have enabled recording and stimulation of electrical activity and provided neuroscientists with a tool for studying cellular signaling processes in complex organs, such as the human brain. ${ }^{16}$ Furthermore, micro- and nanoelectrode arrays have provided possibilities for studying electrochemical signals in cell networks. ${ }^{17-19}$ Nevertheless, establishing a reliable communication between a biological cell and an electrode remains a challenging task partly due to the mechanical mismatch between the soft biological tissues and the rigid electronic chip. $^{20-22}$ An important factor to consider when evaluating electronic interfaces for implants is the Young's modulus of the biological tissue, which lies in the range of $100 \mathrm{~Pa}$ to $10 \mathrm{kPa}$ for tissue of the central nervous system. ${ }^{23-25}$ In contrast, electronic implants exhibit very high elastic moduli in the range of GPa for rigid silicon-based chips. ${ }^{26}$ In vitro, the stiff substrate alters cell shape, organization, and function and therefore does not represent a model close to the natural cellular behavior with soft surrounding tissue. ${ }^{27-30}$ In vivo, the stiff synthetic substrate may trigger inflammatory response or loss of functionalities, indicating rejection of the electronic interface and thus precluding successful translation of these probes to clinical research. ${ }^{20,2331,32}$ Furthermore, rigid electronic implants can alter the physiological movement of organs such as the heart ${ }^{33-35}$ and neuronal tissue. ${ }^{36-38}$ Therefore, numerous studies have been conducted to optimize flexibility and geometrical structure of different substrates for future implants. ${ }^{39-46}$ However, adding electronic functionality to soft substrate materials remains difficult due to technical limitations arising from standard fabrication methods. Recently, bioactive coating of MEAs using hydrogels has been introduced in an effort to overcome the mechanical mismatch of the metal-biological interface. ${ }^{47-51}$ Adding a soft hydrogel layer to neuronal implants significantly decreases the local strain and modulates the immune response in the brain. ${ }^{52,53}$ Likewise, several methods have been investigated to fabricate bioelectronic interfaces on flexible substrates such as polyimide ${ }^{6,54}$ and parylene $e^{42,55}$, or to transfer a metallic pattern onto soft polydimethylsiloxane (PDMS) substrates. ${ }^{56,57}$ Very recently, an implanted neural MEA interface has been developed, which is capable of restoring voluntary control of locomotion after traumatic spinal cord injury. ${ }^{58}$ Moreover, an MEA with platinum-silicone electrodes has been patterned on a PDMS substrate and used for successful in vivo recordings from the spinal cord of a rat. ${ }^{44}$ One of the advantages of using flexible MEAs is to have a conformal contact between the living tissue and the electrode with minimal invasiveness. ${ }^{44}$ Nevertheless, current microfabrication strategies typically require expensive instrumentation as well as time-consuming research and development cycles for each substrate. In addition, most of the flexible substrates and common electrode materials that are compatible with classical fabrication approaches are still relatively stiff compared to the interfacing tissue (see Table 1). This calls for

\footnotetext{
${ }^{1}$ Institute of Bioelectronics (ICS-8) and JARA-Fundamentals of Future Information Technology, Forschungszentrum Jülich, Jülich 52425, Germany and ${ }^{2}$ Neuroelectronics - Munich School of Bioengineering, Department of Electrical and Computer Engineering, Technical University of Munich, Boltzmannstraße 11, D-85749 Garching, Germany Correspondence: Bernhard Wolfrum (bernhard.wolfrum@tum.de)
}

Received: 13 December 2017 Revised: 3 April 2018 Accepted: 4 April 2018

Published online: 24 May 2018 
Table 1. Summary of the elastic modulus of flexible substrates, electrodes, and tissue

\begin{tabular}{lll}
\hline Material & Elastic modulus (MPa) & Ref. \\
\hline Flex substrate & & 101 \\
Parylene-C & $3.2 \times 10^{3}$ & 102 \\
Polyimide & $3 \times 10^{3}$ & 103 \\
SU-8 & $2.2 \times 10^{3}$ & 104 \\
Silk & $19-22$ & 100 \\
PDMS & \\
Agarose & & 105 \\
Gelatin & 2.5 & 106 \\
Electrode & $2.5-4.3 \times 10^{-1}$ & \\
Iridium & $8.1 \times 10^{-2}$ & 107 \\
Platinum & & 107 \\
Gold & $5.3 \times 10^{5}$ & 108 \\
Glassy carbon & $2.5 \times 10^{5}$ & 109 \\
PEDOT:PSS & $7.8 \times 10^{4}$ & 110 \\
Printed carbon composite & $6.2 \times 10^{4}$ & 111 \\
Tissue & $\approx 9 \times 10^{1}-3 \times 10^{2}$ & \\
Heart & $\approx 1$ & 112 \\
Retina & & 113 \\
Gray matter & $1 \times 10^{-2}-5 \times 10^{-1}$ & 25 \\
White matter & $2 \times 10^{-2}$ & 25 \\
Skin & $4.5 \times 10^{-4}$ & 114 \\
\hline a Base:cure ratio of 10:1 & $3 \times 10^{-4}$ & \\
b 2.5\% agarose gel & $0.1-2.4$ & \\
\hline & & \\
\hline
\end{tabular}

technologies that can add electronic functionality to truly soft substrates, such as hydrogels, in a rapid prototyping approach.

Ink-jet printing has recently emerged as a versatile alternative fabrication tool for patterning high-resolution microstructures with complex electrode geometries on the micrometer scale. ${ }^{59-69}$ A major advantage of the fabrication using ink-jet printing is the possibility of changing the structure design in flight. Ink-jet printing eliminates the need for pre-patterned lithographic masks and thus allows for the adaptation of different geometries in a cost- and time-efficient manner. Another advantage of this method is the ease of incorporating newly emerging ink materials such as carbon or PEDOT: PSS (poly(3,4-ethylenedioxythiophene) doped with poly(4-styrenesulfonate)), ${ }^{70}$ which could serve as a better electrode material compared to standard noble metals for cellular interfaces. ${ }^{71}$ In this work, we use a carbon nanoparticle ink to print the interfacing electrode material due to its electrochemical stability, wide electrochemical water window, and low impedance for electrical sensing and stimulation of cellular activity. ${ }^{72-79}$ We shed light on the process required for ink-jet printing high-resolution MEAs, feedlines, and passivation layers on a soft substrate. Functional mircoelectrode arrays are printed on PDMS, agarose, and even gelatin-based substrates including candies (gummy bears). Additionally, we introduce a printed hydrogel MEA for cell recordings, which is challenging to directly pattern using classical photolithographic methods. We demonstrate the functionality of the MEA by extracellular recording of action potentials from $\mathrm{HL}-1$ cells.

\section{RESULTS AND DISCUSSION}

Printed soft MEA arrays

The overall design and fabrication process of the printed PDMS MEAs are shown in Fig. 1. The schematic presents the design of a line MEA (Fig. 1a-c) and the subsequent printing of inks on a PDMS substrate (Fig. 1d-f). Developing functional electronics on soft materials requires the printing of electrically continuous lines. Therefore, it is important that adjacent ink droplets, which are deposited on the substrate, are connected to a functional entity. However, this is typically difficult to achieve with a water-based ink containing the functional material and a hydrophobic substrate such as PDMS. Our pristine PDMS substrates exhibited a static contact angle of $109^{\circ} \pm 3$ with a surface energy of $25 \pm$ $4 \mathrm{mN} / \mathrm{m}$, which is consistent with observations reported in the literature. $^{80}$ The high contact angle consequently leads to a breakup of the deposited liquid structure due to dewetting, effectively causing discrete islands of printed liquid similar to condensed water droplets on a cold surface. One way to avoid dewetting of ink on PDMS surfaces is to implement a multilevel matrix deposition method, in which few drops separated by a defined distance in the $X$ - and $Y$-direction are printed each time until a whole film is completed. This method has been adapted to print on a wide variety of substrates as reported previously. ${ }^{81,82}$ Another way to overcome the dewetting problem is to increase the substrate surface energy via oxygen plasma exposure. ${ }^{83}$ Although the commonly employed method of oxygen plasma treatment does improve the wettability of PDMS it causes spontaneous cracking of the PDMS surface. Upon drying of the printed film, this causes microscopic cracks and consequently failure of the feedline connection. To circumvent this problem, we investigated the alternative of modifying the PDMS surface using (3-mercaptopropyl) trimethoxysilane (MPTMS), which has recently evolved for improving the surface wettability as well as enhancing the adhesion of the deposited ink and preventing crack formation. ${ }^{82,84}$ MPTMS has two different functional groups at its terminals: a methoxy $\left(-\mathrm{OCH}_{3}\right)$ group, which binds to the PDMS and a thiol $(-\mathrm{SH})$ group, which changes the surface properties of the pristine PDMS. ${ }^{57,82,85}$ Here, we modulate the wetting degree of PDMS to pattern large $(>1 \mathrm{~mm})$ and small $(<0.03 \mathrm{~mm})$ structures required for developing MEA. In order to fine tune the PDMS surface energy to meet the requirements of our printing resolution, we controlled the degree of surface modification using MPTMS by changing the incubation time. Figure $2 a$ shows the printing results of carbon ink on the PDMS substrate with different MPTMS incubation using the same line pattern. As seen in Fig. 2b, the water contact angle decreases with longer incubation time. Optimal conditions for continuously formed lines are observed after approximately $60 \mathrm{~min}$ corresponding to a contact angle of $\sim 80^{\circ}$. Larger features can be patterned on PDMS by further increasing the wettability of the surface. Thus, a wide range of structures ranging from high-resolution individual lines of $30 \mu \mathrm{m}$ up to a several millimeter patches can be printed by tuning the MPTMS incubation time.

Fundamentally, the microfabrication of electronic devices for applications in wet environments requires the patterning of a dielectric material, which serves as a passivation layer. In the case of MEA chips for electrophysiological or electrochemical recordings, the passivation layer must be compatible with the cells and provide insulation of the feedlines versus conducting electrolytes. Moreover, it needs to be chemically resistant against the medium supporting the cell culture. For this reason, we chose a polyimide insulation material, which has already demonstrated good passivation properties in clean-room fabricated MEA arrays. ${ }^{86}$ As we are aiming for a fully printed device, it is crucial to choose a suitable ink and to understand possible factors that could induce passivation failure. One common problem in printing continuous passivation films is dewetting, which is especially important for covering areas that are larger than two printed drops. ${ }^{87}$ Considerable work has been reported in the literature to avoid dewetting when printing films either by using algorithmgenerated spacings or by radically changing the surface energy of the substrate. ${ }^{8,89}$ As we discussed earlier, one can tune the 
a)

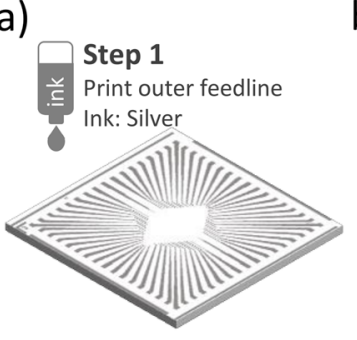

d)

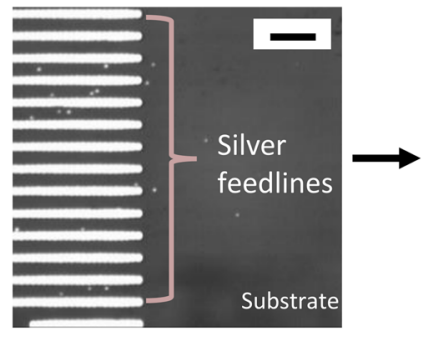

b)

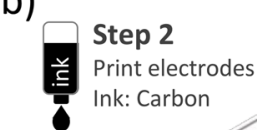

c)

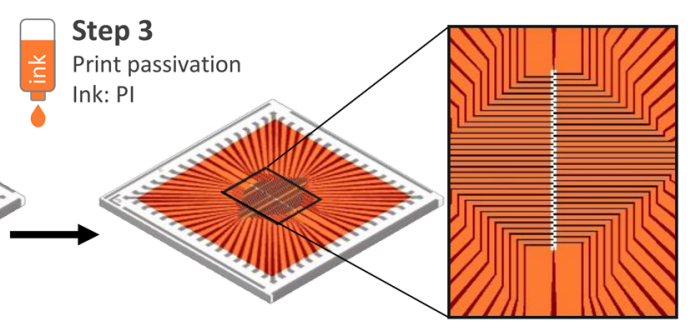

e)

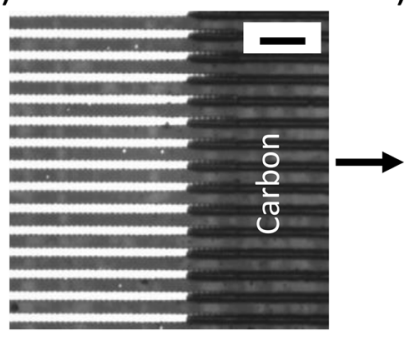

f)

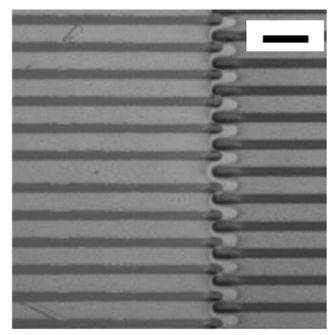

g)
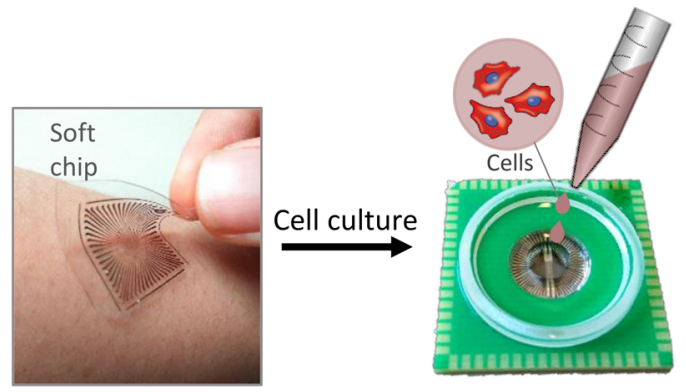

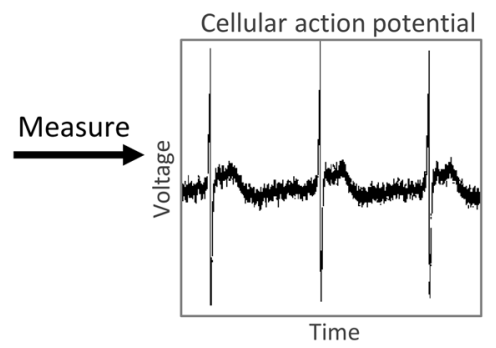

Fig. 1 Sketch of the device principle and printing procedure. a Step 1: outer feedlines are printed with a silver nanoparticle ink on a 12× $12 \mathrm{~mm}^{2}$ substrate. b Step 2: inner feedlines and MEAs are printed with carbon nanoparticle ink. c Step 3: A $9 \times 9 \mathrm{~mm}^{2}$ passivation layer is printed with polyimide ink (PI). d-f Microscopic images of the successive printing process of a carbon MEA on PDMS subsequently depositing d silver ink, e carbon ink, and f PI ink. Scale bars represent $200 \mu \mathrm{m}$. g Principle of the recording of action potentials from electrogenic cells using the printed soft MEA

PDMS wetting degree for water-based inks to a desired state by controlling the incubation time with MPTMS. However, it is not possible to combine two different wetting states: one for printing fine structures while the other enables the printing of large-area films. Obviously, there is no universal wetting state that can meet all the requirements for printing small and large structures with different inks in a single pass. Thus, the development of printed multilayer arrays with carbon MEAs and passivation films requires a change in the surface energy of the PDMS between the individual printing steps. This change has to match the requirements for optimal wetting in order to form a continuous passivation film. We have recently shown that the MPTMS treatment acts as a protective layer and prevents spontaneous crack formation upon plasma treatment. ${ }^{84}$ Here, we took advantage of the chemical modification conducted prior to printing the MEAs and investigated the influence of the plasma dose on the wetting of the passivation ink on PDMS surfaces (see Fig. 2d). This way it was possible to print the passivation layer in a controlled way covering the feedlines without compromising the MEAs or the bond pads for connecting the headstage. The design and fabrication process of our PDMS MEA are shown in Fig. 1. The passivation was printed to expose only a small carbon MEA to the liquid as seen in Fig. 1f. The average width of a single MEA was 30 $\pm 1.5 \mu \mathrm{m}(n=30)$ with a center-to-center distance of $40 \pm 1.5 \mu \mathrm{m}$ $(n=30)$. Details on the characterization of the printed MEA, including electrical, electrochemical, and mechanical evaluation of the device can be found in the Supplementary Information (Figures S1 and S2).

Extracellular recordings using PDMS and hydrogel MEAs

In order to evaluate the functionality of the soft MEA, we performed extracellular recordings of cardiomyocyte-like HL1 cells $^{90}$ (Fig. 3). To this end, the bond pads of the printed PDMS MEAs (Fig. 3a) where electrically connected to a carrier board (Fig. $3 b)$. Cells were cultured on the MEAs until a confluent cell layer developed and evaluated for viability using fluorescent live-dead staining (Fig. 3c). After a few days in culture, the $\mathrm{HL}-1$ cells were spontaneously contracting, confirming the compatibility of the printed devices with the active cell layer. We used the PDMS MEAs to locally monitor action potential generation within the cell culture. Figure $3 d$ shows an example of the electrical signals recorded on different electrodes on the same PDMS MEA. The HL1 cells generated spontaneous action potentials. The maximum cell signal amplitude recorded was $906 \mu \mathrm{V}_{\mathrm{pp}}$ at a background noise of about $62 \mu \mathrm{V}_{\mathrm{pp}}$, which is comparable to reported values of $\mathrm{HL}-1$ recordings using gold MEAs on polyimide substrates, ${ }^{68}$ as well as advanced clean-room-fabricated cell interfaces such as nanocavity electrodes ${ }^{91}$ and nanopillar electrodes ${ }^{92}$ on ceramic substrates.

To show the versatility of our approach for developing MEA structures on soft materials, we investigated printed MEAs on hydrogel substrates such as agarose, gelatin, and edible gummy 
a)

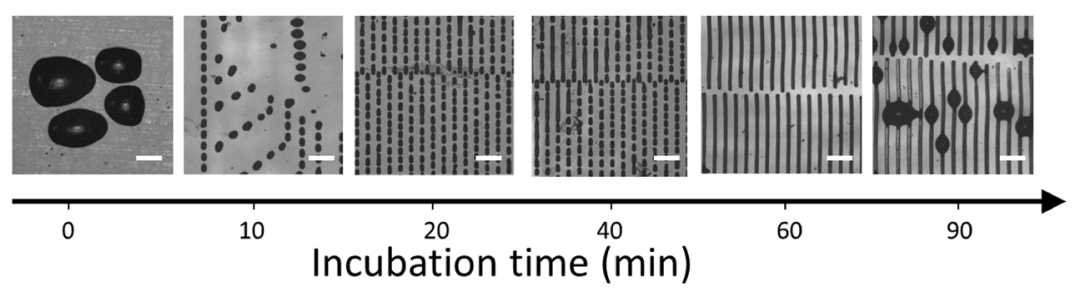

b)

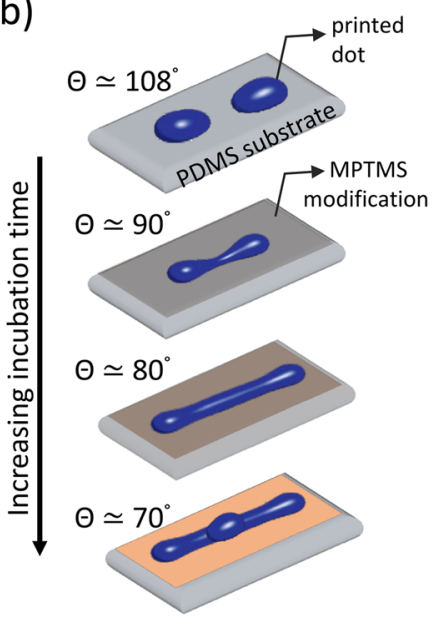

c)

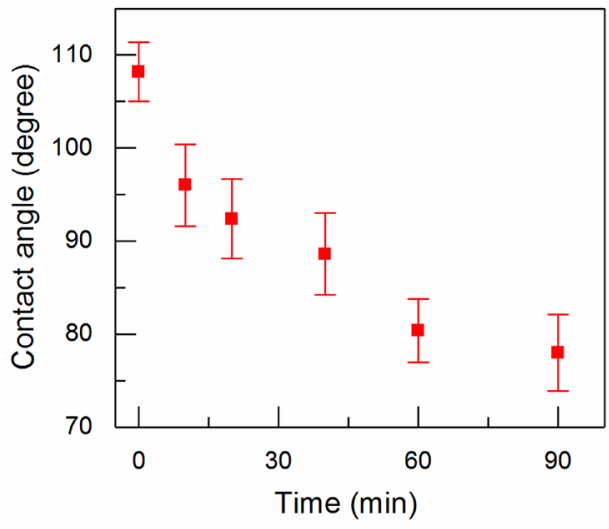

d)

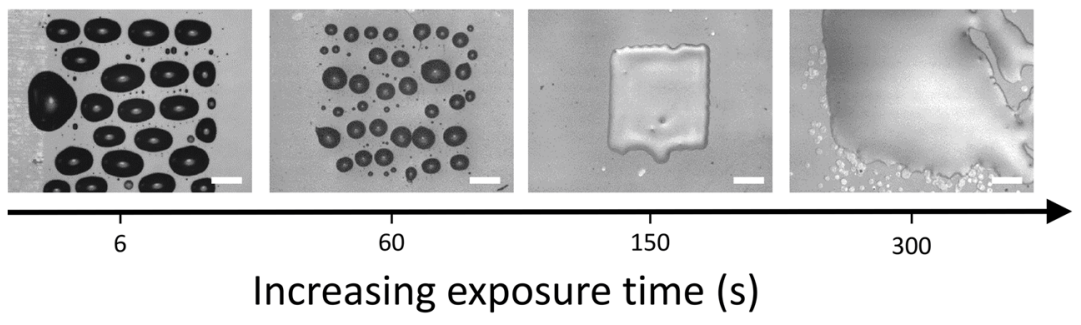

Fig. 2 Effect of MPTMS incubation on printed line formation. a Microscopic images of printed carbon lines with a fixed drop spacing of $20 \mu \mathrm{m}$ versus the incubation time of MPTMS, scale bars represent $200 \mu \mathrm{m}$. b Schematic drawing of the ink spreading on a PDMS substrate. c Measured contact angles of a water drop as a function of the incubation time of MPTMS. $\mathbf{d}$ Optical microscopy images of printed PI ink on PDMS as a function of oxygen plasma exposure time. The design of the structure to be printed was a rectangular shape as shown in the third image of this sequence. All structures were printed with a fixed DPI (dots per inch) of 846. Scale bars represent $200 \mu \mathrm{m}$

bears (see Fig. 4). The functionality of the hydrogel MEAs was evaluated by performing cellular recording of action potentials. Cells were plated on a gelatin-based gummy bear MEA and after a few days in culture, electrical recordings were performed. Figure 4e shows five exemplary traces from the same MEA. We observed a maximum amplitude of $442 \mu \mathrm{V}_{\mathrm{pp}}$ at background noise of approximately $80 \mu \mathrm{V}_{\mathrm{pp}}$ on the hydrogel MEA.

To further examine the specificity of the recorded signals, the cells were chemically stimulated with noradrenaline (NA), a catecholamine that triggers a sympathetic response. As expected, the firing rate of the spontaneous action potential was increased from $1.3 \mathrm{~Hz}$ up to $1.8 \mathrm{~Hz}$ upon addition of $4 \mu \mathrm{L}$ of a $10 \mathrm{mM} \mathrm{NA}$ solution (Fig. 4e). We have chosen gelatin as a substrate for bioelectronic interfaces for several reasons. First, it is a soft material with a Young's modulus in the range of $10^{\circ}-10^{2} \mathrm{kPa} .^{93}$ Second, it has been used as a scaffold for tissue engineering and shown to be a promising material for repairing traumatic injuries to the brain as it improves the brain-tissue reconstruction. ${ }^{94}$ Third, it exhibits antibacterial and hemostatic effects, ${ }^{95,96}$ which is beneficial for recovery after mechanically inserting the electrodes to the desired tissue location. ${ }^{97,98}$ Thus, it can be expected that adding functionality to gelatin-based devices will have an impact on future implant technology. We believe that our approach of printing MEA structures on hydrogel materials such as agarose and gelatin will provide opportunities for developing soft as well as low-cost and disposable functional devices. Furthermore, the concept can be applied in future work for the development of dense multilayer MEA arrays to meet requirements for neuroscience applications in vitro and in vivo.

\section{CONCLUSIONS}

In this work, we demonstrated the development and application of printed MEA arrays on soft substrates including PDMS and hydrogels. To this end, we introduced a straightforward printing process, which exploits controlled wetting properties of carbon and polyimide inks on PDMS, overcoming major problems that typically arise in printing structures at different spatial scales. We presented a printed hydrogel MEA for bioelectronic applications. The soft MEAs were applied for localized recordings of action potentials from $\mathrm{HL}-1$ cells, validating the suitability of the printed devices for electrophysiological measurements. This work represents an important step toward the design of soft hydrogel-based bioelectronic devices using ink-jet printing. 
a)

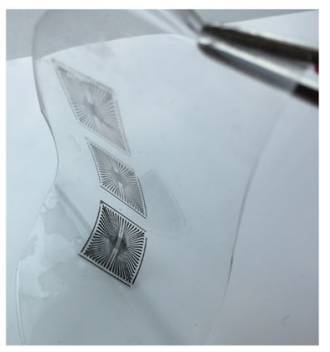

b)

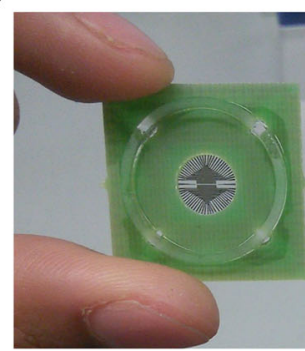

c)

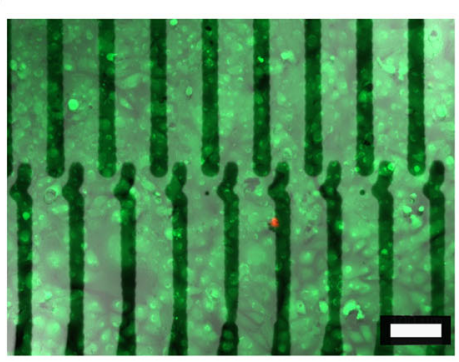

d)

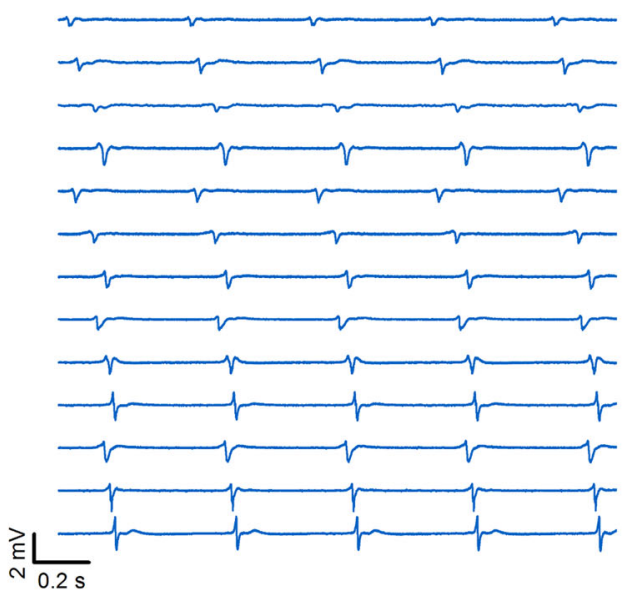

e)

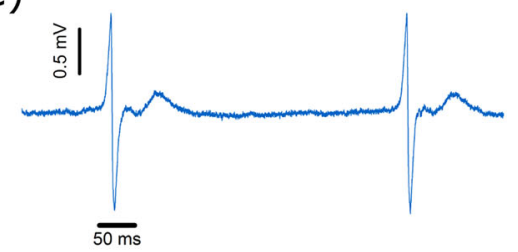

f)

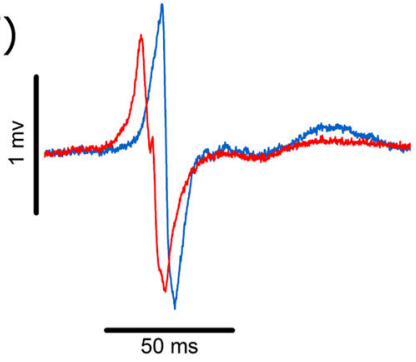

Fig. 3 Final device and demonstration of the printed MEAs on PDMS. a Photograph of printed carbon MEAs on a PDMS substrate. $\mathbf{b}$ Final chip bonded to a printed circuit board and encapsulated for use in cell culture. c Fluorescence microscopy image of live/dead staining of HL-1 cells growing on a PDMS MEA (scale bar $100 \mu \mathrm{m}$ ). Live cells appear green and a single dead cell red. d Action potential recording of different HL-1 cells growing on the same PDMS MEA over a time span of $\sim 2 \mathrm{~s}$. e Magnified display of a single recorded trace. $\mathbf{f}$ Overlay of two individual extracellular recordings. The temporal shift in the two signals indicates the signal propagation across the cell network

We believe that the approach presented in this paper will allow for rapid prototyping of disposable sensor array structures on a variety of soft substrates for in vitro as well as in vivo applications. Potentially, future devices could be directly developed on gelatin taken from an individual and transplanted onto the tissue in the same organism.

\section{METHODS}

Ink-jet printing

MEA arrays were fabricated using an OmniJet 300 ink-jet printer (UniJet Co., Republic of Korea). Silver nanoparticle ink was used for the fabrication of feedlines on PDMS samples (Nano-silver ink DGP 40LT-15C Advanced Nano Products, Co., Ltd, South Korea). The gelatin (gummy bear) and the agarose-based MEAs were printed using carbon ink prepared in our laboratory. Prior to printing, all inks were sonicated for $5 \mathrm{~min}$ and filtered with $0.2 \mu \mathrm{m}$ PVDF syringe filter. Printing was performed using $1 \mathrm{pL} D M C$ cartridges (Fujifilm Dimatix Inc., USA) for printing silver and carbon inks. Ten picoliters cartridges were used for printing the polyimide ink (PIN6400-001; Chisso Corp., Japan) filtered with a $0.2 \mu \mathrm{m}$ PTFE syringe filter. Printing parameters used for printing on all soft substrates were fixed to a jetting frequency of $2 \mathrm{kHz}$, a drop spacing of $20 \mu \mathrm{m}$, a substrate holder and printhead temperature of $25^{\circ} \mathrm{C}$, a jetting period of $16 \mu \mathrm{s}$, and a jetting voltage of $40 \mathrm{~V}$. The distance between the printhead and substrates was set to $250 \mu \mathrm{m}$ for planar substrates. For non-planar substrates (maximum height $3 \mathrm{~mm}$ ) as shown in Fig. 4a, the distance between the printhead and the substrate holder was set to $10 \mathrm{~mm}$. The sintering and curing steps were conducted on a precision hot plate (CT10; Harry Gestigkeit $\mathrm{GmbH}$, Germany) in case of PDMS. In case of gelatin (gummy bears) and agarose gel, samples were cured using a photonic sintering system (PulseForge 1200; NovaCentrix, USA) with six $500 \mu$ s pulses at 100 V. All MEA chips were cut into $12 \mathrm{~mm} \times 12 \mathrm{~mm}$ pieces.

\section{Carbon ink preparation}

The carbon ink was prepared as reported previously. ${ }^{66,67,99}$ Briefly, $1 \mathrm{~g}$ of carbon black (Orion Carbons) in $5 \mathrm{~g}$ of a $50 / 50 \mathrm{wt} \%$ mixture of ethylene glycol and water was milled with $100 \mu \mathrm{m}$ yttrium zirconium beads at $1100 \mathrm{rpm}$ for $1 \mathrm{~h}$ in a Pulverisette 7 ball mill (Fritsch, Germany). The milled mass was diluted with further $10 \mathrm{~g}$ of ethylene glycol/water mixture to adjust the viscosity with vigorous stirring. Next, $0.2 \mathrm{wt} \%$ polyacrylic acid (Sigma Aldrich, $35 \mathrm{wt} \%$ solution) was added to improve the adhesion of the ink to the substrate. Finally, the solution was filtered through a $0.45 \mu \mathrm{m}$ filter to obtain the final ink.

\section{MEA design layout}

As shown schematically in Fig. 1, the multi-electrode microchip consists of 64 individually addressable carbon electrodes with a center-to-center spacing of $40 \pm 1.5 \mu \mathrm{m}$. The size of the final printed chip is $12 \times 12 \mathrm{~mm}$. Figure 1 shows a schematic illustration of the design, the chip, and the printing process on PDMS substrates. In a first step, silver nanoparticle ink is printed to form the feedlines and bond pads of the chip as shown in Fig. 1a. The contact pads are used later for connecting the MEA to the electronic amplifier system. Next, the carbon nanoparticle ink is printed to cover the silver feedline and form an electrode area for cellular measurements (Fig. 1b). This procedure ensures that only carbon is exposed to the electrolyte solution. Finally, the polyimide passivation ink (JNC Corporation, Japan) is printed to cover the chip except for the contact pads and small electrode opening in the center of the chip. For the other substrates, carbon ink was printed directly on the substrate followed by the passivation layer. The final electrode area was $1900 \pm 300 \mu \mathrm{m}^{2}(n=30)$. The average resistance of the carbon feedlines was below $1 \mathrm{k} \Omega$.

\section{Ink characterization}

The particle size of the carbon ink was determined using a scanning electron microscope (SEM-Nova Nano FEl, USA) with an accelerating 


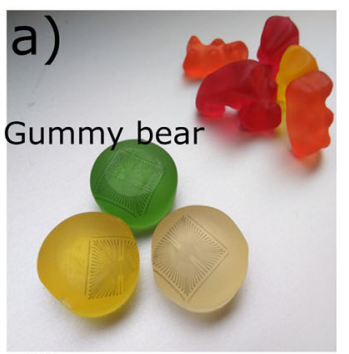

d)

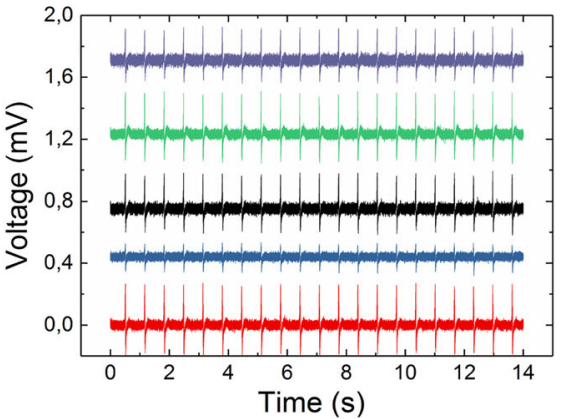

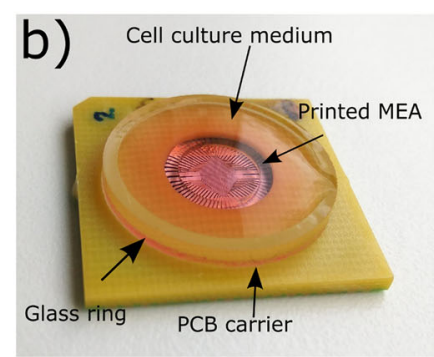

e)

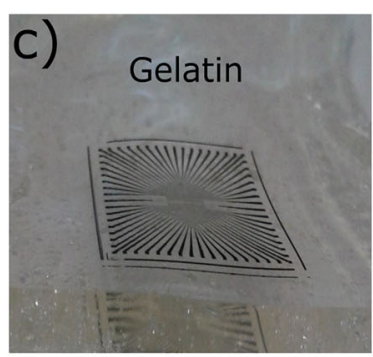

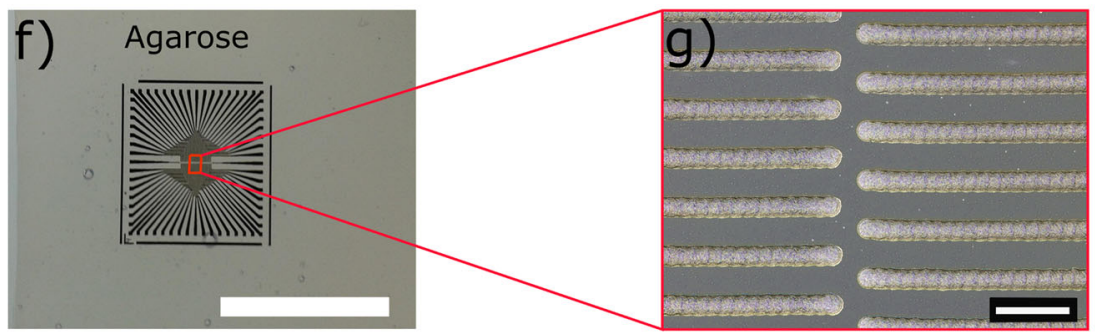

Fig. 4 Printed MEA on soft hydrogel substrates for extracellular recording. a Photograph of printed carbon MEAs on a gummy bear substrate. b Final chip bonded to printed circuit board with HL-1 cells culture. c Exemplary photograph of a printed MEA on a gelatin substrate. d Action potential recording from HL-1 cells using printed carbon MEAs on a gummy bear substrate, traces are offset in y-direction for clarity of representation. e HL-1 cells stimulation with noradrenaline (NA). f Photograph of a printed MEA on an agarose substrate (scale bar $10 \mathrm{~mm}$ ). $\mathbf{g}$ Microscopic image of printed MEA on an agarose substrate (scale bar: $100 \mu \mathrm{m}$ )

voltage of $3 \mathrm{kV}$. We observed nanoparticle diameters in the range of 40-200 nm. The 3-D profile for the printed MEAs was measured using a laser confocal microscope (Keyence VK- X130K) at a wavelength of $658 \mathrm{~nm}$. The height mapping in the $z$-direction revealed a homogeneous topography and a thickness of 500 and $600 \mathrm{~nm}$ for the printed silver and carbon layers, respectively.

The electrical resistance of the printed carbon lines was measured after exposure to a temperature of $120^{\circ} \mathrm{C}$. We performed a four-terminal resistivity measurements yielding a sheet resistance of $47 \pm 12 \Omega / s q(n=$ 10); further details can be found in the Supplementary Information.

\section{PDMS substrate preparation}

Step-1 silicon wafer coating. A silicon wafer (5-inch) was first silanized in order to generate a repellent surface. The coating was achieved by covalently linking perfluorooctyltrichlorosilane (FOTCS) 97\% (from Alfa Aesar) via vapor deposition. First, the wafer was activated in oxygen plasma ( $0.8 \mathrm{mbar}, 3 \mathrm{~min}, 80 \mathrm{~W}$ ) and silanized with FOTCS at $45 \mathrm{mbar}$ for $1.5 \mathrm{~h}$ in an argon atmosphere.

Step-2 PDMS casting. The silicone elastomer PDMS substrates (Sylgard 184 from Dow Corning) were prepared by manually mixing the curing agent and base material at a ratio of 1:10 by mass. This ratio results in a substrate with Young's modulus of $\sim 2.5 \mathrm{MPa}$ as described previously. ${ }^{100}$ In order to cast the elastomer substrate, $10 \mathrm{~mL}$ of the mixture was poured onto a FOTCS-coated silicon wafer and subsequently degassed in a vacuum chamber at room temperature. The PDMS was cured at $60^{\circ} \mathrm{C}$ overnight. Afterwards, the PDMS substrate was easily peeled from the wafer.
Step-3 PDMS surface modification. The surface modification of PDMS substrate was carried out as mentioned previously. ${ }^{84}$ Briefly, PDMS samples were coated by (3-mercaptopropyl) trimethoxysilane (MPTMS 95\%-Sigma Aldrich). To this end, the PDMS substrate was immersed in 1:200 solution of MPTMS in ethanol for $1 \mathrm{~h}$. Afterwards, the sample was rinsed with deionized water (Millipore Milli-Q System, $18 \mathrm{M} \Omega / \mathrm{cm}$ ). Finally, the PDMS samples were immersed in $1 \mathrm{mM} \mathrm{HCl}$ solution for $1 \mathrm{~h}$ and washed again with deionized water. The PDMS substrates were kept in the refrigerator and were used within 2 days.

\section{Oxygen plasma}

An oxygen plasma chamber (100-E plasma system; Technics Plasma $\mathrm{GmbH}$ ) was used for tuning the hydrophilicity of the PDMS surface. The typical dose as used prior to passivation printing was applied exposing the PDMS surface for $150 \mathrm{~s}$ at $40 \mathrm{~W}$ and $0.2 \mathrm{mbar}$ pressure, unless stated otherwise.

Gelatin-based substrate preparation. For the MEAs printed on gummy bear substrates, commercial gummy bears from Haribo ${ }^{\circledR}$ (Haribo $\mathrm{GmbH}$ \& Co. KG, Bonn, Germany) were melted and casted on a silicon wafer. The casted substrate was cleaned by ethanol and washed with deionized water. Finally, the substrate was immersed in deionized water for $8 \mathrm{~h}$ before printing. For the gelatin substrates, $20 \% \mathrm{w} / \mathrm{v}$ gelatin solution was prepared by soaking gelatin powder (Sigma-Aldrich ${ }^{\circledR}$, from porcine skin) in $100 \mathrm{~mL}$ deionized water for $2 \mathrm{~h}$. Next, the solution was heated to approximately $70^{\circ} \mathrm{C}$ until a homogeneous solution was formed. Finally, the warm solution was poured into a Petri dish and allowed to from a gel at room temperature. 
Agarose substrate preparation. Gels were prepared by dissolving $3 \mathrm{~g}$ of agarose (Sigma-Aldrich ${ }^{\circledast}$ ) dissolved in $100 \mathrm{~mL}$ tris/acetate buffer. The gel was poured in Petri dish and kept in the refrigerator. The thickness of the agarose gel was $3.5-4.0 \mathrm{~mm}$.

\section{Contact angle measurement}

The wetting behavior of the PDMS substrate was investigated by contact angle measurement. The sessile drop technique using an OCA H20O instrument (DataPhysics Instruments $\mathrm{GmbH}$ ) was performed at room temperature. A $2 \mu \mathrm{L}$ drop of deionized water was dispensed on top of the PDMS surface. The acquired image of the water on the sample was taken using an integrated camera. The drop profile of a liquid-vapor interface was extracted and fitted by the Young-Laplace function provided by the OCA H200 software. The contact angle at the liquid-solid interface was assigned according to the fitted profile. Measurements were repeated five times for each substrate.

\section{Electrical characterization}

The resistance of the printed test structures was measured using a multimeter (Voltcraft Plus VC 960, Conrad, Germany). The sheet resistance was measured using a four point probe (Jandel CYL-HM21, Bridge Technology, USA).

\section{Electrochemical characterization}

Prior to electrochemical measurements, all chips were cleaned by incubation with ethanol and deionized water for $5 \mathrm{~min}$, each. A glass ring with a height of $10 \mathrm{~mm}$ and a diameter of $7 \mathrm{~mm}$ was glued to the MEAs using PDMS in order to create a reservoir. Electrochemical experiments were performed using a Biological potentiostat (VSP-300 potentiostat from BioLogic Science Instruments). All experiments were carried out in a supporting electrolyte of phosphate-buffered saline (PBS 1×, pH 7.4) using an equimolar mixture of potassium ferricyanide $(1 \mathrm{mM})$ and potassium ferrocyanide (1 mM) (Sigma Aldrich) as a redox tracer dissolved in PBS. The signals were recorded against an $\mathrm{Ag} / \mathrm{AgCl}$ reference electrode (Super Driref SDR 2; World Precision Instruments, USA).

\section{Cellular recording}

Three PDMS MEAs and one gummy-bear-based MEA were sterilized by incubation with $70 \%$ ethanol for $10 \mathrm{~min}$, followed by rinsing with sterile distilled water thrice. They were coated with $2.5 \mu \mathrm{g} / \mathrm{cm}^{2}$ fibronectin from bovine plasma (Sigma Aldrich, Schnelldorf, Germany) in calcium and magnesium free PBS (Life Technologies $\mathrm{GmbH}$, Darmstadt, Germany) at $37^{\circ} \mathrm{C}$ for $1 \mathrm{~h}$. The chips were rinsed once with supplemented Claycomb medium just before cell seeding. Cardiomyocyte-like HL-1 cells were maintained in Claycomb medium (Sigma Aldrich, Steinheim, Germany) supplemented with $10 \mathrm{v} \%$ fetal bovine serum (Life Technologies $\mathrm{GmbH}$, Darmstadt, Germany), $100 \mu \mathrm{g} / \mathrm{ml}$ penicillin-streptomycin (Life Technologies $\mathrm{GmbH}$, Darmstadt, Germany), $0.1 \mathrm{mM}( \pm)$-Norepinephrine (+)-bitartrate salt (Noradrenaline, Sigma Aldrich, Steinheim, Germany) and $2 \mathrm{mM} \mathrm{L-}$ glutamine (Life Technologies $\mathrm{GmbH}$, Darmstadt, Germany) in a humidified incubator at $37^{\circ} \mathrm{C}$ and $5 \% \mathrm{CO}_{2}$. The medium was changed daily. Once confluency was reached, the contracting cell layer was first washed and then detached by incubation with $0.05 \%$ trypsin-EDTA (Life Technologies $\mathrm{GmbH}$, Darmstadt, Germany) at $37^{\circ} \mathrm{C}$. Trypsin digestion was then inhibited by addition of supplemented Claycomb medium and the cells were sedimented by centrifugation at $200 \mathrm{rcf}$ for $5 \mathrm{~min}$. The cells were resuspended in pre-warmed, supplemented Claycomb medium and 100 $\mu \mathrm{L}$ were added to the center of each chip. The cells were left to adhere in a humidified incubator at $37^{\circ} \mathrm{C}$ and $5 \% \mathrm{CO}_{2}$ for $30 \mathrm{~min}$. Afterwards, $500 \mu \mathrm{L}$ of medium were added to each chip. The medium was exchanged daily until confluency was reached. Once the confluent cell layer was beating (after $\sim 2$ days) action potentials were recorded employing a 64 channel MEA amplifier system developed in-house. The system consists of a headstage connected to a main amplifier, which is connected to the controlling PC via a 16-bit A/D converter (USB-6255; National Instruments, Austin, Texas, USA). Data acquisition is controlled through an in-house developed software, which allows the definition of the recording parameters such as gain and filter settings. We limited the effective bandwidth with a bandpass filter from 1 to $3 \mathrm{kHz}$ for all measurements reported.
Data Availability Statement

All experimental data generated or analyzed during this work are included in the article and the Supplementary Information Files.

\section{ACKNOWLEDGEMENTS}

N.A. thanks Hossain Hassani for data analysis. All authors thank Norbert Wolters and Jan Schnitker for help with the amplifier development and acknowledge funding by the Bernstein Center Munich (grant number 01GQ1004A, BMBF). We greatly appreciate the funding from the BCCN (grant number 01GQ1004A, BMBF).

\section{AUTHOR CONTRIBUTIONS}

N.A. developed the printing protocols, fabricated, and characterized all the devices. A. Y. developed the carbon ink. S.S. and S.W. performed the cell experiments. N.A. and B. W. conceived the experiments. N.A. with the help of F.B. and B.W. analyzed the data. N.A. together with B.W. and S.W. wrote the manuscript, which all authors discussed. B. W. and A.O. supervised the work.

\section{ADDITIONAL INFORMATION}

Supplementary Information accompanies the paper on the npj Flexible Electronics website (https://doi.org/10.1038/s41528-018-0027-z).

Competing interests: The authors declare no competing interests.

Publisher's note: Springer Nature remains neutral with regard to jurisdictional claims in published maps and institutional affiliations.

\section{REFERENCES}

1. Berdondini, L. et al. A microelectrode array (MEA) integrated with clustering structures for investigating in vitro neurodynamics in confined interconnected sub-populations of neurons. Sens. Actuators B Chem. 114, 530-541 (2006).

2. Blau, A. et al. Characterization and optimization of microelectrode arrays for in vivo nerve signal recording and stimulation. Biosens. Bioelectron. 12, 883-892 (1997).

3. Hierlemann, A., Frey, U., Hafizovic, S. \& Heer, F. Growing cells atop microelectronic chips: interfacing electrogenic cells in vitro with CMOS-based microelectrode arrays. Proc. IEEE 99, 252-284 (2011).

4. Hong, N., Joo, S. \& Nam, Y. Development of microfluidic channel devices integrated on microelectrode arrays for drug screening with in vitro neuronal culture. In 10th Int. Meet. Substrate-Integrated Microelectrode Arrays 2016 (Reutlingen, Germany, 2016).

5. Stett, A. et al. Biological application of microelectrode arrays in drug discovery and basic research. Anal. Bioanal. Chem. 377, 486-495 (2003).

6. Seo, J.-M. et al. Biocompatibility of polyimide microelectrode array for retinal stimulation. Mater. Sci. Eng. C 24, 185-189 (2004).

7. Moeller, K. D., Uppal, S., Graaf, M., Yeh, N.-H. \& Li, W. Microelectrode arrays and the move toward practical applications. Meet. Abstr. MA2016-02, 3295-3295 (2016).

8. Rutten, W. L. C. Selective electrical interfaces with the nervous system. Annu. Rev. Biomed. Eng. 4, 407-452 (2002).

9. Ludwig, K. A., Uram, J. D., Yang, J., Martin, D. C. \& Kipke, D. R. Chronic neural recordings using silicon microelectrode arrays electrochemically deposited with a poly(3,4-ethylenedioxythiophene) (PEDOT) film. J. Neural Eng. 3, 59 (2006).

10. Kim, E. T. et al. Feasibility of microelectrode array (MEA) based on siliconepolyimide hybrid for retina prosthesis. Invest. Ophthalmol. Vis. Sci. 50, 4337-4341 (2009).

11. Johnstone, A. F. M. et al. Microelectrode arrays: a physiologically based neurotoxicity testing platform for the 21st century. Neurotoxicology 31, 331-350 (2010).

12. Kessler, D. K. The Clarion ${ }^{\circledR}$ Multi-Strategy ${ }^{\mathrm{TM}}$ cochlear implant. Ann. Otol. Rhinol. Laryngol. 108, 8-16 (1999).

13. Clark, G. M. et al. A multiple electrode cochlear implant. J. Laryngol. Otol. 91 935-945 (1977).

14. Chapman, C. A. R., Goshi, N. \& Seker, E. Multifunctional neural interfaces for closed-loop control of neural activity. Adv. Funct. Mater. 28, 1703523 (2017).

15. Wise, K. D., Angell, J. B. \& Starr, A. An integrated-circuit approach to extracellular microelectrodes. IEEE Trans. Biomed. Eng. 17, 238-247 (1970).

16. Wise, K. D. Silicon microsystems for neuroscience and neural prostheses. IEEE Eng. Med. Biol. Mag. 24, 22-29 (2005).

17. Huang, X.-J., O'Mahony, A. M. \& Compton, R. G. Microelectrode arrays for electrochemistry: approaches to fabrication. Small 5, 776-788 (2009). 
18. Sun, G. et al. Ultra-sensitive and wide-dynamic-range sensors based on dense arrays of carbon nanotube tips. Nanoscale 3, 4854-4858 (2011).

19. Yakushenko, A., Kätelhön, E. \& Wolfrum, B. Parallel on-chip analysis of single vesicle neurotransmitter release. Anal. Chem. 85, 5483-5490 (2013).

20. Polikov, V. S., Tresco, P. A. \& Reichert, W. M. Response of brain tissue to chronically implanted neural electrodes. J. Neurosci. Methods 148, 1-18 (2005).

21. Lee, H., Bellamkonda, R. V., Sun, W. \& Levenston, M. E. Biomechanical analysis of silicon microelectrode-induced strain in the brain. J. Neural Eng. 2, 81 (2005).

22. Hoon Lee, J., Kim, H., Hun Kim, J. \& Lee, S.-H. Soft implantable microelectrodes for future medicine: prosthetics, neural signal recording and neuromodulation. Lab. Chip 16, 959-976 (2016).

23. Spedden, E., White, J. D., Naumova, E. N., Kaplan, D. L. \& Staii, C. Elasticity maps of living neurons measured by combined fluorescence and atomic force microscopy. Biophys. J. 103, 868-877 (2012).

24. Saha, K. et al. Substrate modulus directs neural stem cell behavior. Biophys. J. 95 , 4426-4438 (2008).

25. Christ, A. F. et al. Mechanical difference between white and gray matter in the rat cerebellum measured by scanning force microscopy. J. Biomech. 43, 2986-2992 (2010).

26. Fattahi, P., Yang, G., Kim, G. \& Abidian, M. R. A review of organic and inorganic biomaterials for neural interfaces. Adv. Mater. 26, 1846-1885 (2014).

27. Jiang, $X$. et al. Elastic modulus affects the growth and differentiation of neural stem cells. Neural Regen. Res. 10, 1523-1527 (2015).

28. Ali, S., Wall, I. B., Mason, C., Pelling, A. E. \& Veraitch, F. S. The effect of Young's modulus on the neuronal differentiation of mouse embryonic stem cells. Acta Biomater. 25, 253-267 (2015).

29. Hampe, N. et al. Defined 2-D microtissues on soft elastomeric silicone rubber using lift-off epoxy-membranes for biomechanical analyses. Soft Matter 10, 2431-2443 (2014).

30. Ribeiro, A. J. S. et al. Contractility of single cardiomyocytes differentiated from pluripotent stem cells depends on physiological shape and substrate stiffness. Proc. Natl. Acad. Sci. USA 112, 12705-12710 (2015).

31. Turner, J. N. et al. Cerebral astrocyte response to micromachined silicon implants. Exp. Neurol. 156, 33-49 (1999).

32. Lee, H. C. et al. Histological evaluation of flexible neural implants; flexibility limit for reducing the tissue response? J. Neural Eng. 14, 036026 (2017).

33. Carlsson, M. et al. Total heart volume variation throughout the cardiac cycle in humans. Am. J. Physiol. Heart Circ. Physiol. 287, H243-250 (2004).

34. Fleischer, S. \& Dvir, T. Tissue engineering on the nanoscale: lessons from the heart. Curr. Opin. Biotechnol. 24, 664-671 (2013).

35. Feiner, R. \& Dvir, T. Tissue-electronics interfaces: from implantable devices to engineered tissues. Nat. Rev. Mater. 3, 17076 (2018).

36. Gilletti, A. \& Muthuswamy, J. Brain micromotion around implants in the rodent somatosensory cortex. J. Neural Eng. 3, 189 (2006).

37. Kozai, T. D. Y., Jaquins-Gerstl, A. S., Vazquez, A. L., Michael, A. C. \& Cui, X. T. Brain tissue responses to neural implants impact signal sensitivity and intervention strategies. ACS Chem. Neurosci. 6, 48-67 (2014).

38. Karumbaiah, L. et al. The upregulation of specific interleukin (IL) receptor antagonists and paradoxical enhancement of neuronal apoptosis due to electrode induced strain and brain micromotion. Biomaterials 33, 5983-5996 (2012).

39. Rao, L., Zhou, H., Li, T., Li, C. \& Duan, Y. Y. Polyethylene glycol-containing polyurethane hydrogel coatings for improving the biocompatibility of neural electrodes. Acta Biomater. 8, 2233-2242 (2012).

40. Thiébaud, P., Lauer, L., Knoll, W. \& Offenhäusser, A. PDMS device for patterned application of microfluids to neuronal cells arranged by microcontact printing. Biosens. Bioelectron. 17, 87-93 (2002).

41. Guo, L. et al. A PDMS-based integrated stretchable microelectrode array (isMEA) for neural and muscular surface interfacing. IEEE Trans. Biomed. Circuits Syst. 7, 1-10 (2013).

42. Rodger, D. C. et al. Flexible parylene-based multielectrode array technology for high-density neural stimulation and recording. Sens. Actuators B Chem. 132, 449-460 (2008).

43. Lacour, S. P., Courtine, G. \& Guck, J. Materials and technologies for soft implantable neuroprostheses. Nat. Rev. Mater. 1, 16063 (2016).

44. Minev, I. R. et al. Electronic dura mater for long-term multimodal neural interfaces. Science 347, 159-163 (2015).

45. Rivnay, J., Wang, H., Fenno, L., Deisseroth, K. \& Malliaras, G. G. Next-generation probes, particles, and proteins for neural interfacing. Sci. Adv. 3, e1601649 (2017).

46. Someya, T., Bao, Z. \& Malliaras, G. G. The rise of plastic bioelectronics. Nature 540, 379 (2016).

47. Kim, D.-H., Abidian, M. \& Martin, D. C. Conducting polymers grown in hydrogel scaffolds coated on neural prosthetic devices. J. Biomed. Mater. Res. A 71A, 577-585 (2004).
48. Kim, D.-H., Wiler, J. A., Anderson, D. J., Kipke, D. R. \& Martin, D. C. Conducting polymers on hydrogel-coated neural electrode provide sensitive neural recordings in auditory cortex. Acta Biomater. 6, 57-62 (2010).

49. Nyberg, T., Inganäs, O. \& Jerregård, H. Polymer hydrogel microelectrodes for neural communication. Biomed. Microdev. 4, 43-52 (2002).

50. Shi, Y. et al. Nanostructured conductive polypyrrole hydrogels as high-performance, flexible supercapacitor electrodes. J. Mater. Chem. A 2, 6086-6091 (2014).

51. Wirthl, D. et al. Instant tough bonding of hydrogels for soft machines and electronics. Sci. Adv. 3, e1700053 (2017).

52. Kim, D.-H. et al. Soft, Fuzzy, and Bioactive Conducting Polymers for Improving the Chronic Performance of Neural Prosthetic Devices. in Indwelling Neural Implants: Strategies for Contending with the In Vivo Environment (ed. Reichert, W. M.) (Duke University, North Carolina, 2008).

53. Spencer, K. C. et al. Characterization of mechanically matched hydrogel coatings to improve the biocompatibility of neural implants. Sci. Rep. 7, 1952 (2017).

54. Stieglitz, T., Beutel, H., Schuettler, M. \& Meyer, J.-U. Micromachined, polyimidebased devices for flexible neural interfaces. Biomed. Microdev. 2, 283-294 (2000).

55. Lee, W. et al. Transparent, conformable, active multielectrode array using organic electrochemical transistors. Proc. Natl Acad. Sci. USA 114, 201703886 (2017).

56. Meacham, K. W., Giuly, R. J., Guo, L., Hochman, S. \& DeWeerth, S. P. A lithographically-patterned, elastic multi-electrode array for surface stimulation of the spinal cord. Biomed. Microdev. 10, 259-269 (2008).

57. Byun, I., Coleman, A. W. \& Kim, B. Transfer of thin Au films to polydimethylsiloxane (PDMS) with reliable bonding using (3-mercaptopropyl)trimethoxysilane (MPTMS) as a molecular adhesive. J. Micromech. Microeng. 23, 085016 (2013).

58. Capogrosso, M. et al. A brain-spine interface alleviating gait deficits after spinal cord injury in primates. Nature 539, 284-288 (2016).

59. Abbel, R., Teunissen, P., Michels, J. \& Groen, W. A. Narrow conductive structures with high aspect ratios through single-pass inkjet printing and evaporationinduced dewetting. Adv. Eng. Mater. 17, 615-619 (2015).

60. Kang, B. J. \& Oh, J. H. Geometrical characterization of inkjet-printed conductive lines of nanosilver suspensions on a polymer substrate. Thin Solid Films 518, 2890-2896 (2010).

61. Wang, J. Z., Zheng, Z. H., Li, H. W., Huck, W. T. S. \& Sirringhaus, H. Dewetting of conducting polymer inkjet droplets on patterned surfaces. Nat. Mater. 3, 171-176 (2004).

62. Sele, C. W., von Werne, T., Friend, R. H. \& Sirringhaus, H. Lithography-free, selfaligned inkjet printing with sub-hundred-nanometer resolution. Adv. Mater. 17, 997-1001 (2005).

63. Singh, M., Haverinen, H. M., Dhagat, P. \& Jabbour, G. E. Inkjet printing-process and its applications. Adv. Mater. 22, 673-685 (2010).

64. Meier, H., Löffelmann, U., Mager, D., Smith, P. J. \& Korvink, J. G. Inkjet printed, conductive, $25 \mu \mathrm{m}$ wide silver tracks on unstructured polyimide. Phys. Status Solidi A 206, 1626-1630 (2009).

65. Khan, Y. et al. Inkjet-printed flexible gold electrode arrays for bioelectronic interfaces. Adv. Funct. Mater. 26, 1004-1013 (2016).

66. Adly, N. et al. Flexible microgap electrodes by direct inkjet printing for biosensing application. Adv. Biosyst. 1, 3 (2017).

67. Adly, N. Y. et al. Three-dimensional inkjet-printed redox cycling sensor. 7, 5473-5479 (2017).

68. Bachmann, B. et al. All-inkjet printed gold microelectrode arrays for extracellular recording of action potentials. Flex. Printed Electron. 2, 035003 (2017).

69. Wolfrum, B. et al. Nanoscale electrochemical sensor arrays: redox cycling amplification in dual-electrode systems. Acc. Chem. Res. 49, 2031-2040 (2016).

70. Eom, S. H. et al. Polymer solar cells based on inkjet-printed PEDOT:PSS layer. Org. Electron. 10, 536-542 (2009).

71. Khodagholy, D. et al. In vivo recordings of brain activity using organic transistors. Nat. Commun. 4, 1575 (2013).

72. Vomero, M. et al. A novel pattern transfer technique for mounting glassy carbon microelectrodes on polymeric flexible substrates. J. Micromech. Microeng. 26, 025018 (2016)

73. VanDersarl, J. J., Mercanzini, A. \& Renaud, P. Integration of 2D and 3D thin film glassy carbon electrode arrays for electrochemical dopamine sensing in flexible neuroelectronic implants. Adv. Funct. Mater. 25, 78-84 (2015).

74. Banks, C. E. \& Compton, R. G. New electrodes for old: from carbon nanotubes to edge plane pyrolytic graphite. Analyst 131, 15-21 (2006).

75. Kassegne, S. et al. Electrical impedance, electrochemistry, mechanical stiffness, and hardness tunability in glassy carbon MEMS $\mu \mathrm{ECoG}$ electrodes. Microelectron. Eng. 133, 36-44 (2015).

76. Benck, J. D., Pinaud, B. A., Gorlin, Y. \& Jaramillo, T. F. Substrate selection for fundamental studies of electrocatalysts and photoelectrodes: inert potential windows in acidic, neutral, and basic electrolyte. PLOS ONE 9, e107942 (2014). 
77. Castagnola, E. et al. Biologically compatible neural interface to safely couple nanocoated electrodes to the surface of the brain. ACS Nano 7, 3887-3895 (2013).

78. Asplund, M., Nyberg, T. \& Inganäs, O. Electroactive polymers for neural interfaces. Polym. Chem. 1, 1374-1391 (2010).

79. Kotov, N. A. et al. Nanomaterials for Neural Interfaces. Adv. Mater. 21, 3970-4004 (2009).

80. Zhang, H. \& Chiao, M. Anti-fouling coatings of poly(dimethylsiloxane) devices for biological and biomedical applications. J. Med. Biol. Eng. 35, 143-155 (2015).

81. Tekin, E., de Gans, B.-J. \& S. Schubert, U. Ink-jet printing of polymers-from single dots to thin film libraries. J. Mater. Chem. 14, 2627-2632 (2004).

82. Wu, J. et al. Inkjet-printed microelectrodes on PDMS as biosensors for functionalized microfluidic systems. Lab. Chip 15, 690-695 (2015).

83. Lin, J. et al. Surface energy patterning for inkjet printing in device fabrication. in Proc. of SPIE (eds Bao, Z. \& McCulloch, I.) 74171D (The International Society for Optical Engineering, 2009).

84. Adly, N. Y. et al. Observation of chemically protected polydimethylsiloxane: towards crack-free PDMS. Soft Matter 13, 6297-6303 (2017).

85. Singh, J. \& Whitten, J. E. Adsorption of 3-mercaptopropyltrimethoxysilane on silicon oxide surfaces and adsorbate interaction with thermally deposited gold. J. Phys. Chem. C 112, 19088-19096 (2008).

86. Georgiev, A. et al. in Chemical and Physical Properties of Polyimides: Biomedical and Engineering Applications. Vol. 7417 (ed. Mbadie, M. M.) Ch. 4 (InTech, Rijeka, Croatia, 2012).

87. Liimatta, T., Halonen, E., Sillanpää, H., Niittynen, J. \& Mäntysalo, M. Inkjet printing in manufacturing of stretchable interconnects. in 2014 IEEE 64th Electronic Components and Technology Conference (ECTC), Orlando, FL 151-156 (2014).

88. Soltman, D., Smith, B., Kang, H., Morris, S. J. S. \& Subramanian, V. Methodology for inkjet printing of partially wetting films. Langmuir 26, 15686-15693 (2010).

89. Park, H. Y., Kang, B. J., Lee, D. \& Oh, J. H. Control of surface wettability for inkjet printing by combining hydrophobic coating and plasma treatment. Thin Solid Films 546, 162-166 (2013).

90. Claycomb, W. C. et al. HL-1 cells: a cardiac muscle cell line that contracts and retains phenotypic characteristics of the adult cardiomyocyte. Proc. Natl Acad. Sci. USA 95, 2979-2984 (1998).

91. Czeschik, A. et al. Nanostructured cavity devices for extracellular stimulation of HL-1 cells. Nanoscale 7, 9275-9281 (2015).

92. Brüggemann, D. et al. Nanostructured gold microelectrodes for extracellular recording from electrogenic cells. Nanotechnology 22, 265104 (2011).

93. Czerner, M., Fellay, L. S., Suárez, M. P., Frontini, P. M. \& Fasce, L. A. Determination of elastic modulus of gelatin gels by indentation experiments. Procedia Mater. Sci. 8, 287-296 (2015).

94. Zhang, T. et al. Three-dimensional gelatin and gelatin/hyaluronan hydrogel structures for traumatic brain injury. J. Bioact. Compat. Polym. 22, 19-29 (2007).

95. Totre, J., Ickowicz, D. \& Domb, A. J. in Biodegradable Polymers in Clinical Use and Clinical Development (eds Domb, A. J., Kumar, N. \& Ezra, A.) 91-109 (John Wiley \& Sons, Inc., 2011).

96. Sundaram, C. P. \& Keenan, A. C. Evolution of hemostatic agents in surgical practice. Indian J. Urol. 26, 374-378 (2010)

97. Lind, G., Linsmeier, C. E., Thelin, J. \& Schouenborg, J. Gelatine-embedded electrodes-a novel biocompatible vehicle allowing implantation of highly flexible microelectrodes. J. Neural Eng. 7, 046005 (2010).

98. Witteveen, J. A. et al. Gelatin/glycerol coating to preserve mechanically compliant nanowire electrodes from damage during brain implantation. J. Vac. Sci. Technol. B 28, C6K13-C6K16 (2010).

99. Hamad, E. M. et al. Inkjet printing of UV-curable adhesive and dielectric inks for microfluidic devices. Lab. Chip 16, 70-74 (2015).
100. Gray, D. S., Tien, J. \& Chen, C. S. Repositioning of cells by mechanotaxis on surfaces with micropatterned Young's modulus. J. Biomed. Mater. Res. A 66A 605-614 (2003).

101. Noh, H., Moon, K., Cannon, A., Hesketh, P. J. \& Wong, C. P. Wafer bonding using microwave heating of parylene for MEMS packaging. in 2004 Proc. 54th Electronic Components and Technology Conference (IEEE Cat. No. 04CH37546, Las Vegas, NV, USA), Vol. 1, 924-930 (2004).

102. Allen, M. G., Mehregany, M., Howe, R. T. \& Senturia, S. D. Microfabricated structures for the in situ measurement of residual stress, Young's modulus, and ultimate strain of thin films. Appl. Phys. Lett. 51, 241-243 (1987).

103. Gao, J., Guan, L. \& Chu, J. Determining the Young's modulus of SU-8 negative photoresist through tensile testing for MEMS applications. in Proc. of SPIE, 754464 (The International Society for Optical Engineering, Hangzhou, China, 2010).

104. Lawrence, B. D. et al. Effect of hydration on silk film material properties. Macromol. Biosci. 10, 393-403 (2010).

105. Normand, V., Lootens, D. L., Amici, E., Plucknett, K. P. \& Aymard, P. New insight into agarose gel mechanical properties. Biomacromolecules 1, 730-738 (2000).

106. Karimi, A. \& Navidbakhsh, M. Material properties in unconfined compression of gelatin hydrogel for skin tissue engineering applications. Biomed. Tech. (Berl.) 59 479-486 (2014).

107. Dimov, S., Menz, W. \& Fillon, B. 4M 2006-Second International Conference on Multi-Material Micro Manufacture (Elsevier, Grenoble, France, 2006).

108. Wu, B., Heidelberg, A. \& Boland, J. J. Mechanical properties of ultrahigh-strength gold nanowires. Nat. Mater. 4, 525 (2005).

109. Manoharan, M., Lee, H., Rajagopalan, R., Foley, H. \& Haque, M. Elastic properties of 4-6 nm-thick glassy carbon thin films. Nanoscale Res. Lett. 5, 14-19 (2009).

110. ElMahmoudy, M. et al. Tailoring the electrochemical and mechanical properties of PEDOT:PSS films for bioelectronics. Macromol. Mater. Eng. 302, 1600497 (2017).

111. Schlatter, S., Rosset, S. \& Shea, H. Inkjet printing of carbon black electrodes for dielectric elastomer actuators. in Proc. of SPIE - The International Society for Optical Engineering. Vol. 10163, 1016311 (Bellingham, Spie-Int Soc Optical Engineering, Bellingham, Washington, USA, 2017).

112. Huyer, L. D. et al. Biomaterial based cardiac tissue engineering and its applications. Biomed. Mater. Bristol Engl. 10, 034004 (2015).

113. Jones, I. L., Warner, M. \& Stevens, J. D. Mathematical modelling of the elastic properties of retina: a determination of Young's modulus. Eye 6, 556 (1992).

114. Kalra, A., Lowe, A. \& Al-Jumaily, A. M. Mechanical behaviour of skin: a review. J. Mater. Sci. Eng. 5, 2169-0022 (2016).

Open Access This article is licensed under a Creative Commons Attribution 4.0 International License, which permits use, sharing, adaptation, distribution and reproduction in any medium or format, as long as you give appropriate credit to the original author(s) and the source, provide a link to the Creative Commons license, and indicate if changes were made. The images or other third party material in this article are included in the article's Creative Commons license, unless indicated otherwise in a credit line to the material. If material is not included in the article's Creative Commons license and your intended use is not permitted by statutory regulation or exceeds the permitted use, you will need to obtain permission directly from the copyright holder. To view a copy of this license, visit http://creativecommons. org/licenses/by/4.0/.

(c) The Author(s) 2018 